\title{
OUTCOMES WITH OAE AND AABR SCREENING IN THE FIRST 48 HOURS - IMPLICATIONS FOR NEWBORN HEARING SCREENING IN DEVELOPING COUNTRIES
}

\author{
Michelle van Dyk ${ }^{\text {a, }}$ \\ De Wet Swanepoel a, b, c* \\ James W. Hall III ${ }^{\text {a, d }}$ \\ ${ }^{a}$ Department of Speech-Language Pathology and Audiology, University of Pretoria, South Africa \\ ${ }^{b}$ Ear Sciences Centre, School of Surgery, The University of Western Australia, Nedlands, \\ Australia \\ ${ }^{c}$ Ear Science Institute Australia, Subiaco, Australia \\ ${ }^{d}$ Department of Speech, Language, and Hearing Sciences, University of Florida, USA
}

\section{${ }^{*}$ Corresponding author:}

Prof. De Wet Swanepoel

Department of Speech-Language Pathology and Audiology

University of Pretoria, c/o Lynnwood and University Road, Hatfield, South Africa, 0002

Email: dewet.swanepoel@up.ac.za $\quad$ Contact Number: +2712 4204280 


\section{ABSTRACT}

Objective: Early discharge of newborns ( $<24$ hours after birth) from birthing centres is an important barrier to successful newborn hearing screening (NHS) in developing countries. This study evaluated the outcome of NHS within the first 48 hours using an automated auditory brainstem response (AABR) device without the need for costly disposables typically required, and transient evoked otoacoustic emissions (TEOAE).

Methods: NHS was performed on one hundred and fifty healthy newborns (300 ears) with TEOAE and AABR techniques before discharge at a hospital. A three-stage screening protocol was implemented consisting of an initial screen with TEOAE (GSI AUDIOscreener+) and AABR (Beraphone MB 11). Infants were screened at several time points as early as possible after birth. Infants were only re-screened if either screening technique (TEOAE or AABR) initially yielded a refer outcome. The same audiologist performed all TEOAE and AABR screenings.

Results: Over the three-stage screen AABR had a significantly lower refer rate of $16.7 \%$ (24/144 subjects) compared to TEOAE (37.9\%; 55/145 subjects). Screening refer rate showed a progressive decrease with increasing age. For both TEOAE and AABR, refer rate per ear screened 24 hours post birth was significantly lower than for those screened before 24 hours. For infants screened before 12 hours post birth, the AABR refer rate per ear (51.1\%) was significantly lower than the TEOAE refer rate (68.9\%). Overall AABR refer rate per ear was similar for infants screened between 24 to 36 hours $(20.2 \%)$ and 36 to 48 hours $(18.9 \%)$ but significantly lower than for TEOAE (40.7\% and $41.9 \%$, respectively). Lowest initial refer rates per ear (TEOAE $25.8 \%$, AABR $3.2 \%$ ) were obtained after 48 hours post birth.

Conclusion: In light of the early post birth discharge typical in developing countries like South Africa, in-hospital screening with AABR technology is significantly more effective than TEOAEs. AABR screening with a device like the MB 11 is particularly appropriate because disposable costs are negligible. 


\section{KEYWORDS:}

Age at screen; Developing countries; Early intervention; Newborn hearing screening;

Otoacoustic emissions; Automated Auditory Brainstem Response

\section{Introduction}

Prevalence of congenital and early-onset hearing impairment ranges from 0.5 to 5 per 1000 infants based on studies from various countries [1-6]. At least $90 \%$ of infants with hearing loss live in developing countries [7]. Undetected hearing loss can lead to delayed or impaired speech and language development, social and emotional problems, academic failure and restricted vocational outcomes [8-11]. The earlier a hearing loss is detected, the earlier intervention can begin, which increases the likelihood of optimizing a child's potential across developmental areas $[2,10]$.

It is recommended that universal newborn hearing screening (UNHS) be performed within the first month of life, and that a screen result be obtained before hospital discharge whenever possible to reduce the subsequent need for outpatient follow-up [11]. All infants should have access to hearing screening during which a physiologic measure such as otoacoustic emissions (OAE) or automated auditory brainstem responses (AABR) [11] is used. Although both AABR and OAE are accepted as reliable measures for newborn hearing screening (NHS) they may present with false-positive results due to patient and environment related factors [12]. AABR is less affected 24 to 48 hours post birth than OAE by transient conditions in the external auditory canal (e.g. collapse of the ear canal and the presence of debris) and middle ear (e.g. presence of amniotic fluid and mesenchyme), making it more likely that newborns will refer with OAE screening than AABR screening [13,14]. Environmental factors such as excessive ambient noise in the test environment or test skills and experience of the screening staff may also negatively affect screening outcomes for both OAE and AABR [15]. Falsepositive results may lead to parental anxiety and worry as well as monetary costs resulting from parents' lost time from work, transportation to health care facilitates, 
unnecessary tests, and probably more consequential costs and follow-up defaults which is a matter of special concern in developing countries like South Africa [16,17].

The recommended time for NHS screening after birth is later than 24 hours to avoid the increased incidence of transient outer and middle-ear conditions affecting screening outcomes in the first hours post birth $[9,15]$. Screening with an OAE technique within the first 24 hours post birth reportedly results in referral rates as high as $20 \%[9,18,19]$. Referral rates drop to as low as 3\% when screening is performed between 24 and 48 hours after birth $[9,18,19]$. Referral rates of less than $4 \%$ are generally achievable when an infant is screened with OAE combined with AABR in a two-step screening system or with $A A B R$ alone before discharge $[15,20]$.

The reported distribution of typical discharge times for newborns in the United Kingdom are $16 \%$ on the day of birth, $35 \%$ the following day; $21 \%$ after 2 days and $28 \%$ for 3 days after delivery [21]. In the US, healthy infants are typically discharged from the hospital between 24 and 48 hours after birth [22]. In comparison healthy infants in South Africa are discharged from a state hospital or clinics between 6 and 24 hours after birth $[23,24]$. Postnatal care is provided by family members or at primary health care clinics [25], even though the World Health Organization [26] recommends that newborns born in health facilities should not be sent home in the crucial first 24 hours of life.

Early discharge of newborns in South Africa is an important challenge to successful implementation of hospital-based NHS. An additional challenge is the cost associated with screening, particularly costs related to disposables involved in testing each infant. Typically AABR screening has been more expensive than OAE screening due to the higher costs of disposables [27]. In South Africa the vast majority (81\%) of private hospitals conducting screening reportedly use OAE screening in the healthy newborn ward compared to only $1 \%$ employing $A A B R$, due to the additional costs associated with this type of screening [28]. The AABR's higher specificity reduces the costs of further diagnostic testing, however, as well as the time parents have to invest in order to reach 
a diagnosis [27]. In South Africa, only $53 \%$ of private hospitals reported some form of NHS, due to lack of appropriate equipment and time constraints [28].

AABR screening is rare in the public health sector of South Africa due to the significantly increased costs compared to $O A E$ screening. AABR equipment is typically more costly than OAE screening [29]. However, it is the increased disposable-related expense of $A A B R$ (e.g., disposable ear tips or muffs and electrodes) that raise the costs significantly. A newer generation AABR device, the Beraphone MB 11 (Maico), has provided an alternative $A A B R$ tool without the requirement for disposables. Its design eliminates the need for disposable ear tips and electrodes, allowing for AABR screening at significantly reduced costs per screen [30]. This type of technology may allow screening of infants at early ages in a health care context where babies are typically discharged before 24 hours after birth, without the costs associated with traditional AABR equipment. Screening technology with limited disposable costs, and that is less susceptible to transient middle ear influences within the first 48 hours after birth, may more readily be utilized for hospital-based screening in typical developing world contexts like the South African public health care system. The aim of this study was therefore to evaluate the outcome of NHS within the first 48 hours using the MB 11 AABR device compared to transient evoked otoacoustic emissions (TEOAE) screening.

\section{Methods}

Newborn hearing screening was conducted in a hospital in South Africa. Institutional research and ethics committee approval was obtained from the University of Pretoria and the hospital involved before data collection commenced.

\subsection{Subjects}

Hearing screening with TEOAE and AABR was performed before hospital discharge for one hundred and fifty healthy newborns (300 ears). Infants were screened at several 
points in time as early as possible after birth. Delays in obtaining informed consent due to hospital protocol, time of delivery, and other logistical factors resulted in some delays to screening. All newborns participating in the study had no documented medical difficulties and were in a well-baby nursery. There were 75 male (50\%) and 75 female $(50 \%)$ infants. The median gestational age was 39 weeks and the mean birth weight was 3208 grams (SD 396 grams). The majority of newborns were born via caesarean section $(74.2 \%)$, which is representative of births in the private health care sector in South Africa.

A pilot study with TEOAE and AABR screening techniques was conducted on sixty healthy newborns before the formal data collection phase commenced. This allowed the audiologist to refine screening techniques, test procedures, and data collection before commencing the study.

\subsection{Screening Protocol}

All parents of infants to be screened were provided with an information brochure prior to screening. Screening was conducted either in a room within the maternity ward or in the nursery, depending on the space available. After informed consent was obtained from a parent, each newborn underwent screening with the TEOAE and AABR. Infants were screened at several points in time as early as possible after birth. Infants were only rescreened if either of the screening techniques (OAE or $A A B R$ ) initially yielded a refer outcome. All TEOAE and AABR screening was performed by the same audiologist. The audiologist was experienced in NHS.

A three-stage screening protocol (figure 1 ) with the TEOAE and AABR was implemented. A refer outcome in the first stage indicated that further screening was required before discharge, to rule out any uncertainty regarding the hearing status of the infant. Refer criterion for subjects was a unilateral or bilateral refer for either screening device. 


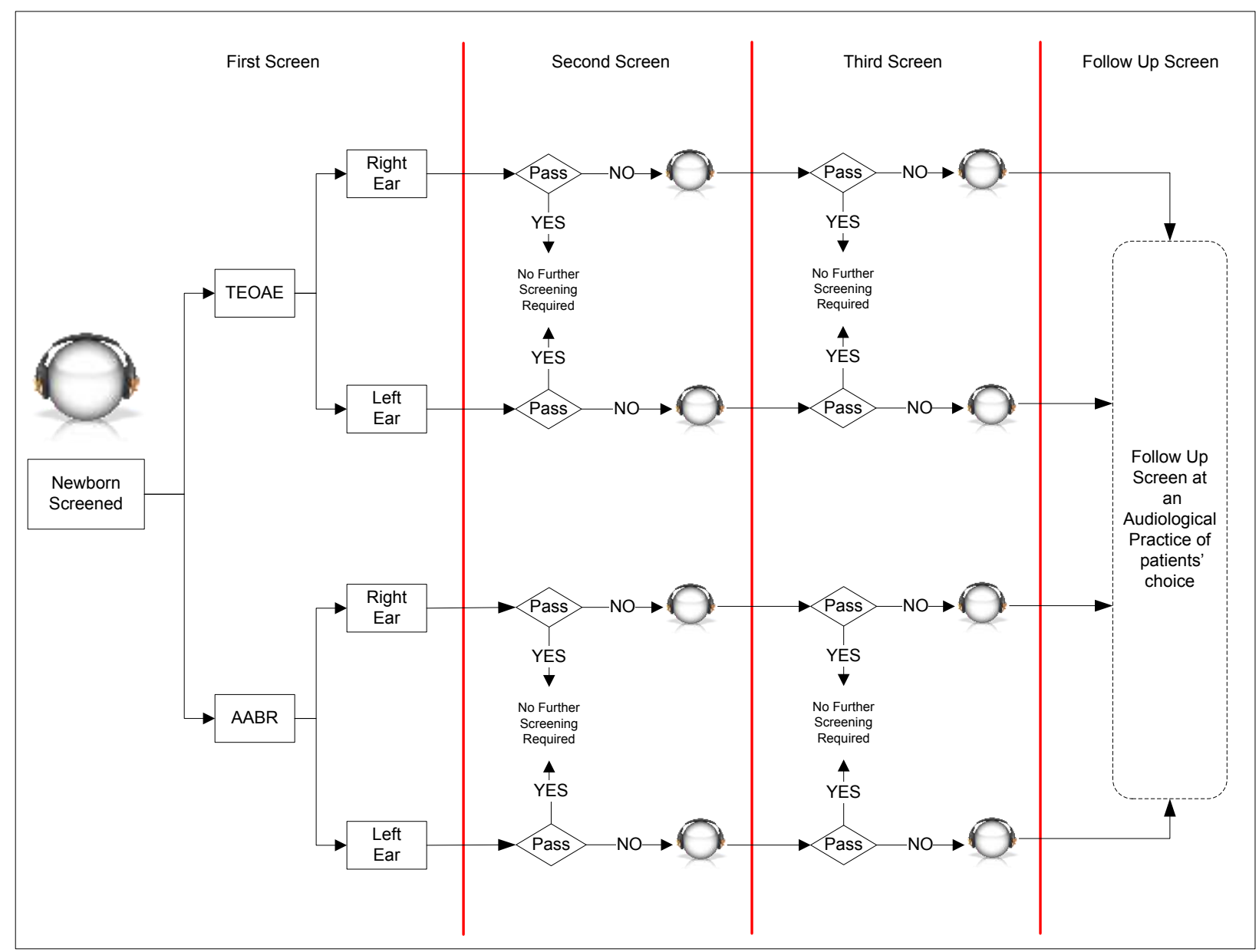

Figure 1. Three-Stage screening protocol employed during data collection

A second-stage screen with the equipment (TEOAE or AABR) was only conducted on ears that yielded a refer result during the initial screen. The third-stage screen was also conducted in the same manner. If a newborn did not pass the third-stage screen, an opportunity was provided for an appointment for a re-screen at the hospital between 2 days and 6 weeks after birth. A screen was not repeated within a stage unless the environment was too noisy or incorrect placement/insertion was evident. Ғoo noisy" was defined by the noise parameters set on either the TEOAE or AABR, and a placement/insert problem was identified when the calibration of either screening technique was unsuccessful. The first ear to be screened was randomly selected, depending on which ear was most accessible (i.e., facing upwards away from the cot) before the infant was turned over to screen the opposite ear. TEOAE screening was 
conducted first $83.1 \%$ of time, while AABR was conducted first $16.9 \%$ of the time. This was due to equipment-related factors established during the pilot study. The cold gel tended to wake babies if $A A B R$ screening was conducted first, and the gel was difficult to remove before conducting the TEOAE. The TEOAE was less invasive with regard to preparation of the newborn and, thus, had less effect on the newborn's state for the next screening method. Test time was recorded for each screening test, excluding the time required to set up, start up and shut down each screening device and to prepare the infant. The ear specific time segments were measured with a stopwatch for the TEOAE and read from the MB 11 software for the AABR screening.

\subsection{Instrumentation}

The screening techniques provided a pass or refer result without the need for a subjective data analysis. The Beraphone MB 11 AABR device (figure 2) consists of a



Figure 2. Beraphone MB 11 screening an infant

handheld headphone unit that integrates the preamplifier and a set of three fixed touchelectrodes connected to a laptop computer [30]. Electrode gel was applied at the three electrode sites (vertex, ground and mastoid) on the baby's head. Electrode placement was as follows: non-inverting electrode was placed on the vertex; inverting electrode on the mastoid ipsilateral to the stimulus; and ground electrode was positioned just above 
the ear ipsilateral to the stimulus. The vertex electrode could be adjusted to the individual size of the baby's head. Electrode gel was placed on the integrated electrodes before the earphone was placed surrounding the baby's ear with the resting electrodes on the prepared sites. The CE-Chirp stimulus ${ }^{\mathrm{TM}}$ was presented at $93 \mathrm{CE}-$ chirps ${ }^{\circledR}$ per second at $35 \mathrm{~dB} \mathrm{nHL}$. Results were reported as either a pass or refer. The result was a pass if the presence of a non-random signal was detected with a confidence level $>99.9 \%$ within 120 seconds. The result was a refer if pass criterion was not reached [30,31]. The cut-off frequencies of the band pass filter were $163 \mathrm{~Hz}$ and $1930 \mathrm{~Hz}[32]$.

TEOAE screening was conducted using the GSI AUDIOscreener+ ${ }^{\mathrm{TM}}$. The probe of this handheld device was placed in the external ear canal of the newborn with a rubber tip. The device used in-ear calibration before screening commenced. The click stimulus intensity was set at $84 \mathrm{~dB}$ peak equivalent SPL at a rate of $64 \mathrm{~Hz}$ for a maximum time of 240 seconds (band pass filter of 1000 to $4000 \mathrm{~Hz}$ ). An automated pass criterion of two bands was utilized based on TEOAE signal to noise ratio (max. noise $60 \mathrm{~dB}$ and max. signal $70 \mathrm{~dB}$ ) and TEOAE reproducibility within 128 to 2048 frames. The TEOAE frequency band low cut-offs were 3500,2500 , and 1500 . The high cut-offs were 4500 , 3500 and 2500 . A reproducibility value of 60 to $80 \%$ was required for the band response to be considered a pass.

\subsection{Data management and analysis}

All data were recorded and subsequently captured on an MS Excel database. SPSS version 21 was used for the statistical analysis. Descriptive statistics provided the frequency distribution and measures of central tendency. Chi-square test was used to investigate correspondence between test outcomes. Analysis of differences in outcomes across ages was performed by grouping three age categories and conducting the Wilcoxon signed rank test and Mann-Whitney test. The significance level for all statistical tests was set at the $5 \%$ level. 


\section{Results}

Initial TEOAE and AABR screening was completed on 150 healthy newborns (300 ears) at various ages post birth.

\subsection{Screening outcomes}

As summarized in Table 1, most ears were successfully screened. A small number of ears were not screened due to the infants' state, noise levels, and/or probe fit issues. Only one ear $(1 / 300 ; 0.3 \%)$ could not be screened with either the TEOAE or AABR throughout the three-stage screen, and $92.7 \%$ of ears (278/300) were screened with both TEOAE and AABR techniques initially. $41.3 \%$ of subjects passed bilaterally with both TEOAE and AABR at the initial screen. Over the three-stage screen TEOAE had a significantly higher refer rate of $37.9 \%$ (55/145 subjects) than AABR (16.7\%; $24 / 144$ subjects). Overall AABR had a significantly ( $p<0.001$; Chi-Square) lower initial refer rate per ear compared to the TEOAE. Right ears had a significantly $(p<0.05$; Chi-Square) lower refer rate for both screening techniques compared to left ears. Rescreen refer rates were also higher per ear for TEOAE (49.5\%) compared to AABR (36.1\%) screening (Table 2). The TEOAE presented with a higher false-positive (i.e. an ear referred initially but passed on the second or third screen) rate $(39 / 103 ; 37.9 \%)$ than the $\operatorname{AABR}(3 / 61 ; 4.9 \%)$.

Mean screen duration for a pass result was 31 seconds (SD 26) for TEOAE and 53 seconds (SD 40) for the AABR. The mean duration for a refer result was 109 seconds (SD 18) with TEOAE and always 180 seconds for AABR due to the test protocol. If the pass criterion was not reached after 180 seconds of test time, the result -efer" was displayed in the lower right corner. There was no significant difference $(p>0.05$; Wilcoxon) in time between the left and right ears when both passed or both referred with a TEOAE. Half the TEOAE pass results (48.5\%) were obtained within the first 20 seconds of screening and half the AABR pass results (50.0\%) were obtained between 11 and 40 seconds. 
Table 1. Outcomes of three-stage newborn hearing screening with TEOAE and AABR

\begin{tabular}{|c|c|c|c|c|}
\hline & \multicolumn{2}{|l|}{ TEOAE } & \multicolumn{2}{|l|}{ AABR } \\
\hline & $\mathbf{N}$ (ears) & $\%$ & $\mathbf{N}$ (ears) & $\%$ \\
\hline \multicolumn{5}{|l|}{ FIRST SCREEN } \\
\hline Refer rate right & $56 / 146$ & 38.4 & $27 / 145$ & 18.6 \\
\hline Refer rate left & $66 / 143$ & 46.2 & $39 / 145$ & 26.9 \\
\hline Refer rate combined & $122 / 289$ & 42.2 & $66 / 290$ & 22.8 \\
\hline Unable to screen & $11 / 300$ & 3.7 & $10 / 300$ & 3.3 \\
\hline \multicolumn{5}{|l|}{ SECOND SCREEN } \\
\hline Refer rate right & $21 / 43$ & 48.8 & $14 / 25$ & 56.0 \\
\hline Refer rate left & $24 / 46$ & 52.2 & $10 / 29$ & 34.5 \\
\hline Refer rate combined & $45 / 89$ & 50.6 & $24 / 54$ & 44.4 \\
\hline Unable to screen & $3 / 92$ & 3.3 & & \\
\hline \multicolumn{5}{|l|}{ THIRD SCREEN } \\
\hline Refer rate right & $3 / 7$ & 42.9 & $1 / 5$ & 20.0 \\
\hline Refer rate left & $3 / 7$ & 42.9 & & \\
\hline Refer rate combined & $6 / 14$ & 42.9 & $1 / 7$ & 14.3 \\
\hline Unable to screen & & & $1 / 8$ & 12.5 \\
\hline \multicolumn{5}{|l|}{ OVERALL SCREEN } \\
\hline Refer rate right & $31 / 146$ & 21.2 & $12 / 145$ & 8.3 \\
\hline Refer rate left & $39 / 143$ & 27.3 & $18 / 145$ & 12.4 \\
\hline Refer rate combined & $70 / 289$ & 24.2 & $30 / 290$ & 10.3 \\
\hline Unable to screen & $11 / 300$ & 3.7 & $10 / 300$ & 3.3 \\
\hline
\end{tabular}


Table 2. Screening outcomes before $\mathbf{2 4}$ hours and after $\mathbf{2 4}$ hours post birth

\begin{tabular}{|c|c|c|c|c|c|c|}
\hline & \multicolumn{2}{|c|}{ FIRST SCREEN } & \multicolumn{2}{|c|}{ SECOND SCREEN } & \multicolumn{2}{|c|}{ THIRD SCREEN } \\
\hline & $\mathbf{N}$ (ears) & $\%$ & $\mathbf{N}$ (ears) & $\%$ & $\mathbf{N}$ (ears) & $\%$ \\
\hline \multicolumn{7}{|l|}{$<12$ hours } \\
\hline TEOAE refer rate right & $16 / 23$ & 69.6 & & & & \\
\hline TEOAE refer rate left & $15 / 22$ & 68.2 & & & & \\
\hline TEOAE refer rate combined & $31 / 45$ & 68.9 & & & & \\
\hline AABR refer rate right & $10 / 24$ & 41.7 & & & & \\
\hline$A A B R$ refer rate left & $14 / 23$ & 60.9 & & & & \\
\hline$A A B R$ refer rate combined & $24 / 47$ & 51.1 & & & & \\
\hline \multicolumn{7}{|l|}{ 12-24 hours } \\
\hline TEOAE refer rate right & $17 / 39$ & 43.6 & $2 / 5$ & 40.0 & & \\
\hline TEOAE refer rate left & $18 / 36$ & 50.0 & $1 / 4$ & 25.0 & & \\
\hline TEOAE refer rate combined & $35 / 75$ & 46.7 & $3 / 9$ & 33.3 & & \\
\hline AABR refer rate right & $8 / 40$ & 20.0 & $1 / 4$ & 25.0 & & \\
\hline$A A B R$ refer rate left & $11 / 35$ & 31.4 & $4 / 5$ & 80.0 & & \\
\hline$A A B R$ refer rate combined & $19 / 75$ & 25.3 & $5 / 9$ & 55.6 & & \\
\hline \multicolumn{7}{|l|}{ 24-36 hours } \\
\hline TEOAE refer rate right & $13 / 42$ & 31.0 & $8 / 12$ & 66.7 & & \\
\hline TEOAE refer rate left & $16 / 42$ & 38.1 & $6 / 11$ & 54.5 & & \\
\hline TEOAE refer rate combined & $29 / 84$ & 34.5 & $14 / 23$ & 60.9 & & \\
\hline$A A B R$ refer rate right & $4 / 38$ & 10.5 & $5 / 8$ & 62.5 & & \\
\hline$A A B R$ refer rate left & $6 / 40$ & 15.0 & $4 / 8$ & 50.0 & & \\
\hline$A A B R$ refer rate combined & $10 / 78$ & 12.8 & $9 / 16$ & 56.3 & & \\
\hline \multicolumn{7}{|l|}{ 36-48 hours } \\
\hline TEOAE refer rate right & $9 / 28$ & 32.1 & $4 / 8$ & 50.0 & $2 / 5$ & 40.0 \\
\hline TEOAE refer rate left & $11 / 26$ & 42.3 & $7 / 14$ & 50.0 & $1 / 3$ & 33.3 \\
\hline TEOAE refer rate combined & $20 / 54$ & 37.0 & $11 / 22$ & 50.0 & $3 / 8$ & 37.5 \\
\hline AABR refer rate right & $5 / 30$ & 16.7 & $2 / 5$ & 40.0 & & \\
\hline AABR refer rate left & $7 / 29$ & 24.1 & & & & \\
\hline AABR refer rate combined & $12 / 59$ & 20.3 & $2 / 11$ & 18.2 & & \\
\hline \multicolumn{7}{|l|}{$<48$ hours } \\
\hline TEOAE refer rate right & $3 / 15$ & 20.0 & $7 / 18$ & 38.9 & $1 / 2$ & 50.0 \\
\hline TEOAE refer rate left & $5 / 16$ & 31.3 & $10 / 17$ & 58.8 & $2 / 3$ & 66.7 \\
\hline TEOAE refer rate combined & $8 / 31$ & 25.8 & $17 / 35$ & 48.6 & $3 / 5$ & 60.0 \\
\hline AABR refer rate right & & & $3 / 8$ & 37.5 & & \\
\hline$A A B R$ refer rate left & $1 / 18$ & 5.6 & $2 / 10$ & 20.0 & $1 / 2$ & 50.0 \\
\hline AABR refer rate combined & $1 / 31$ & 3.2 & $5 / 18$ & 27.8 & $1 / 3$ & 33.3 \\
\hline
\end{tabular}




\subsection{Age effect on screening outcome}

Screening refer rate per ear showed a progressive decrease with increasing age (Figure $3)$. The AABR refer rate per ear was significantly lower $(p<0.001$; Chi-Square) than the

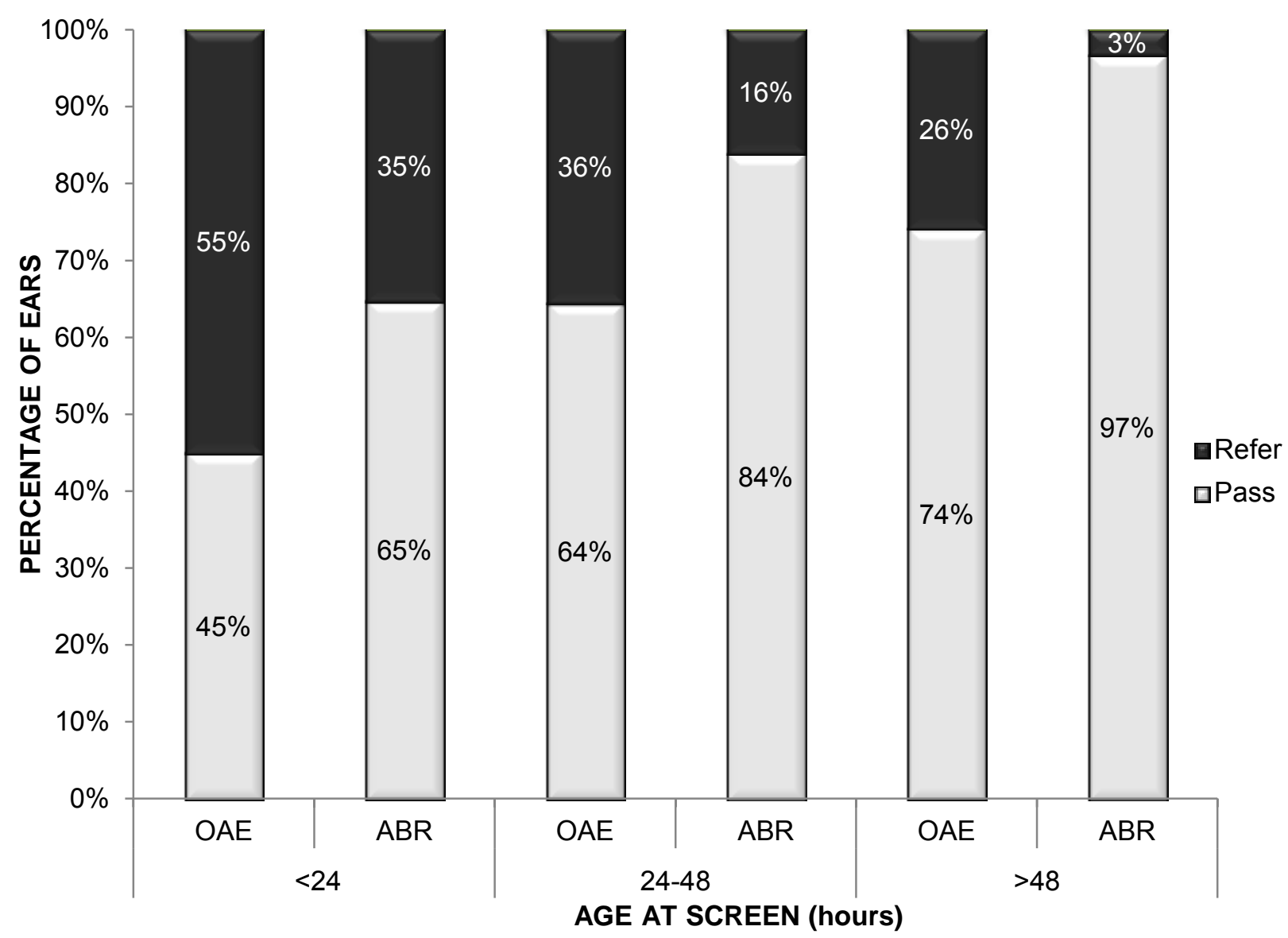

Figure 3. Initial screening outcomes according to age at screen (TEOAE $\mathbf{n = 2 8 9}$ ears; AABR $n=290$ ears)

TEOAE refer rate when an infant was screened before 12 hours after birth. Overall TEOAE refer rate per ear was similar for infants screened between 24 and 36 hours (40.7\%) and between 36 and 48 hours (41.9\%). Overall AABR refer rate per ear for infants screened between 24 and 36 hours $(20.2 \%)$ and between 36 and 48 hours (18.9\%) was also similar but significantly lower than for the TEOAE. Lowest initial refer rates per ear and per subject (TEOAE 35.3\%, AABR 5.6\%) were obtained after 48 
hours post birth (Average age for TEOAE, 61 hours post birth; average age for AABR, 57 hours post birth). As indicated in Figure 3, the refer rate for ears screened after 24 hours was significantly $(p<0.001$; Chi-square) less than those screened before 24 hours for both AABR and TEOAE. The majority of infants were screened between 24 and 48 hours (TEOAE 47.8\%, AABR 47.2\%). The percentage of infants screened before 24 hours post birth was $41.5 \%$ with TEOAE and $42.1 \%$ with AABR. Few of the infants were screened 48 hours post birth for both screening techniques (TEOAE 10.7\%; AABR $10.7 \%)$.

The mean age for a pass result with the TEOAE during the first screen was 32 hours (SD 15) and 25 hours (SD 14) for a refer result. The mean age for an AABR pass result was 31 hours (SD 15) and 22 hours (SD 13) for a refer result. Mean age at screen was significantly greater for those with a pass result compared to those with a refer result with either the AABR or TEOAE ( $p<0.05$; Mann-Whitney test).

\section{Discussion}

Scheduling timing of newborn hearing screening beyond 48 hours, or even 24 hours, post birth to avoid excessive referral rates is a challenge in developing countries like South Africa where healthy newborns are typically discharged from 6 hours after birth [33]. Even though AABR is typically less affected by transient conductive pathology than OAE screening, it has not been widely adopted in existing newborn screening programs in South Africa $[30,34]$. This has primarily been attributed to the increased costs related to screening due to the disposables typically required for AABR screening as opposed to $\mathrm{OAE}$ [27]. Although the AABR technique may involve a slightly higher initial equipment cost than TEOAE technique, a newer generation $A A B R$ (the MB 11 by BERAphone) reduces screening costs and newborn preparation time because disposable electrodes and ear couplers are not required [35-37].

Consistent with the findings of previous studies [36,38], we found that referral rate decreased progressively with increasing age for TEOAE and AABR. Screening with 
AABR reduced referral rates significantly compared to TEOAE regardless of age at screen. AABR also had a lower rescreen refer rate than TEOAE. Overall subject referral (after initial and rescreen) using TEOAE was more than twice that of AABR. Refer rate for ears screened with either AABR or TEOAE after 24 hours was significantly less than those screened before 24 hours. Although AABR refer rate per ear improved with increasing age, slightly more than half of the infants yielded a refer outcome within 12 hours post birth and approximately one-quarter of the infants referred when screened between 12 and 24 hours after birth.

Transient conductive auditory dysfunction negatively influences screening results in newborns, leading to a significantly increased probability of a refer result [19]. In public hospitals in South Africa infants may be discharged within 24 hours post birth when TEOAE refer rate is highest $[36,39]$. The constraint of birthing facility discharge typically from 6 hours after birth for healthy babies and their mothers may necessitate the introduction of an initial or second-stage screening with $A A B R$ to minimize the referral rates prior to diagnostic evaluation $[15,38]$. In this study newborns initially screened with AABR at 48 hours or later had the optimal subject refer rate of $5.6 \%$ when compared to the recommended benchmark of less than 4\% [11]. Excessive referral rates place an additional burden on NHS program resources (i.e., screening costs) and negatively influence successful tracking and follow-up of referred infants [40].

The risk of high TEOAE referral rates before 48 hours post birth, as demonstrated in this study, make it difficult to overlook initial AABR screening even in a resourceconstrained environment like South Africa [15]. However, OAE screening techniques are typically the most widely used for initial or two-stage NHS programs worldwide, including in South Africa. The apparent explanation is a perception that OAE screening is easier and quicker to perform with less expense related to consumables than the AABR $[14,15,28,41,42]$. Reported overall TEOAE referral rates for subjects from NHS programs in developing countries vary considerably from those for our study (37.9\%) with referral rates of $33.2 \%$ reported for Nigeria, $30 \%$ in Brazil, and $10.5 \%$ in Turkey $[7,19,37]$. The overall AABR screen referral rate per subject in this study $(16.7 \%)$ was 
higher than $A A B R$ MB 11 screening programs reported from other countries such as India (9.1\%), Germany (3.8\%) and Turkey (2\%) [35,37,43]. A number of factors contribute to the higher refer rate in our study apart from the fact that this study was not an evaluation of an existing NHS program. The test environment in this study was neither a separate dedicated room nor a sound treated room, and screening was often conducted in the nursery $[19,37]$. Another contributing factor could be the high caesarean delivery rate compared to subjects in previously reported studies where the caesarean delivery rate was less than 15\% [42,44]. Most importantly however the higher average refer rate for both TEOAE and AABR is largely due to the large number of ears screened within 24 hours post birth. Other studies typically screened primarily before hospital discharge but at least 48 hours post birth $[7,35,37,43]$.

Initial screening with $A A B R$ significantly reduces the number of infants that require follow-up retesting outside of hospital discharge even for those younger than 48 hours post birth. In developing countries like South Africa where most newborns are discharged before 24 hours after birth, OAE screening is not ideal $[36,45,46]$. AABR is therefore recommended for NHS screening for these younger children. An added advantage of $A A B R$ is the possibility of detection of auditory neuropathy spectrum disorder typically missed by OAE screening [11]. Birthing facilities typically plagued with resource constraints related to disposable-related costs could benefit from an AABR device like the MB11 that does not require disposables. Ideally, however, newborns should be screened as late post birth as possible with best results evident after 48 hours post birth. If a hospital is unable to screen a newborn from 24 hours after birth before discharge alternative screening contexts in developing countries like South Africa may need to be considered such as immunization clinics and Midwife Obstetric Units (MOUs) in order to reduce high referral rates and the risk of excessive follow-up defaults $[47,48]$. 


\section{Conclusion}

Initial screening with an AABR technology (MB 11 BERAphone ${ }^{\circledR}$ ) is significantly more effective than TEOAE for newborns younger than 48 hours. Screening infants within 24 hours post birth with $A A B R$ results in reduced costs associated with high referral and false-positive rates. In view of the early discharge typical in South Africa and other developing countries, AABR screening using technology without disposable-related costs may be the most appropriate choice for sustainable and cost-effective programs. However, even AABR may not be an entirely efficient option for birthing centres where infants are discharged within 24 hours after birth, due to high referral rates which influence factors such as costs, logistics, infrastructural considerations, case definition, targeted referral rates and follow-up default [15]. UNHS protocols for contexts like the South African public health care sector may require AABR technology (without the burden of disposable-related costs) in hospital-based settings with OAE reserved for screening older infants at health care visits, such as community-based immunization clinics or midwife obstetric units $[14,46,47]$. Utilizing different cost-effective technologies in various health contexts relating to infant age may be essential to ensure that such screening programs in developing countries like South Africa are successful.

\section{References}

[1] B.O. Olusanya, Highlights of the new WHO report on newborn and infant hearing screening and implications for developing countries, Int. J. Pediatr. Otorhinolaryngol. 75 (2011) 745-748.

[2] World Health Organization, Neonatal and infant hearing screening, Current issues and guiding principles for action, Outcome of a WHO informal consultation held at WHO head-quarters, Geneva, Switzerland, 9-10 November, 2009, Geneva, WHO, 2010.

[3] B.O. Olusanya, V.E. Newton, Global burden of childhood hearing impairment and disease control priorities for developing countries, The Lancet 369 (2007) 1314-1317. 
[4] J. Attias, M. Al-Masri, L. Abukader, G. Cohen, P. Merlov, H. Pratt, R. OthmanJebara, P. Aber, F. Raad, A. Noyek, The prevalence of congenital and early-onset hearing loss in Jordanian and Israeli infants: La Incidencia de hipoacusias congénitas y de inicio temprano en niños jordanos e israelíes, Int. J. Aud. 45 (2006) 528-536.

[5] A. Parving, The need for universal neonatal hearing screening-some aspects of epidemiology and identification, Acta Paediatrica, 88 (2001) 69-72. doi: 10.1111/j.16512227.1999.tb01163.x

[6] D.C. Thompson, H. McPhillips, R.L. Davis, T.A. Lieu, C.J. Homer, M. Helfand, Universal newborn hearing screening: summary of evidence, Jama 286 (2001) 20002010.

[7] B.O. Olusanya, A. Emokpae, J.K. Renner, S.L. Wirz, Costs and performance of early hearing detection programs in Lagos, Nigeria, Trans. R. Soc. Trop. Med. Hyg. 103 (2009) 179-186.

[8] M.P. Moeller, Early intervention and language development in children who are deaf and hard of hearing, Pediatrics 106 (2000) 43-51.

[9] A. Erenberg, J. Lemons, C. Sia, D. Trunkel, P. Ziring, Newborn and infant hearing loss: detection and intervention, American Academy of Pediatrics, Task Force on Newborn and Infant Hearing, 1998-1999, Pediatrics 103 (1999) 527-530.

[10] C. Yoshinaga-Itano, A.L. Sedey, D.K. Coulter, A.L. Mehl, Language of early-and later-identified children with hearing loss, Pediatrics 102 (1998) 1161-1171.

[11] Joint Committee on Infant Hearing $(\mathrm{JClH})$, Joint Committee on Infant Hearing Year 2007 Position Statement: principles and guidelines for early hearing detection and intervention programs, Pediatrics 120 (2007) 898-921. 
[12] A.L. Mehl, V. Thomson, The Colorado newborn hearing screening project, 19921999: on the threshold of effective population-based universal newborn hearing screening, Pediatrics 109 (2002) E7.

[13] S.A. Gabbard, J.L. Northern, C. Yoshinaga-Itano, Hearing screening in newborns under 24 hours of age, In Seminars in hearing, Thieme Medical Publishers, Inc., vol. 20 (1999) 291-304.

[14] O.V. Akinpelu, E. Peleva, W.R.J. Funnell, S.J. Daniel. Otoacoustic emissions in newborn hearing screening: A systematic review of the effects of different protocols on test outcomes, Int. J. Pediatr. Otorhinolaryngol. 78 (2014) 711-717.

[15] B.O. Olusanya, B.A. Bamigboye, Is discordance in TEOAE and AABR outcomes predictable in newborns, Int. J. Pediatr. Otorhinolaryngol. 74 (2010) 1303-1309.

[16] F.H. Bess, J. Gravel, Foundations of pediatric audiology: identification and assessment. Plural Pub Inc, 2006.

[17] E. Fitzpatrick, I. D. Graham, A. Durieux-Smith, D. Angus, D. Coyle, Parents' perspectives on the impact of the early diagnosis of childhood hearing loss: Perspectiva de los padres sobre el impacto del diagnóstico temprano en la hipoacusia de la infancia, Int. J. Aud. 46 (2007) 97-106.

[18] S.G. Korres, D.G. Balatsouras, T. Nikolopoulos, G.S. Korres, E. Ferekidis, Making universal newborn hearing screening a success, Int. J. Pediatr. Otorhinolaryngol. 70 (2006) 241-246.

[19] L.D.M. Lupoli, L. Garcia, A.R.T Anastasio, A.C. Fontana, Time after birth in relation to failure rate in newborn hearing screening, Int. J. Pediatr. Otorhinolaryngol. 77 (2013) 932-935. 
[20] A.L. Mehl, V. Thomson, Newborn hearing screening: the great omission, Pediatrics 101 (1998) 4-9.

[21] A. Elattar, E.M. Selamat, A.A. Robson, A.D. Loughney, Factors influencing maternal length of stay after giving birth in a UK hospital and the impact of those factors on bed occupancy, J. Obstetrics. Gynaecol. 28 (2008) $73-76$.

[22] Southeast Georgia Health System, http://www.sghs.org/body.cfm?id=158 (accessed on 29.07.11).

[23] Government Communication and Information System (GCIS), a National Government Department of the Republic of South Africa, as represented by the Chief Executive Officer of the GCIS, http://www.services.gov.za»HomesServices for residents (accessed on 30.07.11).

[24] Mowbray Maternity Hospital, http://mowbraymaternity.co.za/index.php/maternalcare.html, 2011 (accessed 21 February 2014).

[25] R.N. Ngunyulu, F.M. Mulaudzi, Indigenous Practices Regarding Postnatal Care at Sikhunyani Village in Limpopo province of South Africa, Afr. J. Nurs. Midwifery 11 (2009) 48-64.

[26] World Health Organization, Postnatal Care, http://www.who.int/maternal child adolescent/topics/newborn/postnatal care/en/, 2014 (accessed 19 February 2014).

[27] H.C. Boshuizen, G.J. Van der Lem, M.A. Kauffman-de Boer, G.A. Van Zanten, A.M. Oudesluys-Murphy, P.H. Verkerk, Costs of different strategies for neonatal hearing screening: a modelling approach. Archives of Dis. in Child. Fetal and Neonatal Ed. 85 (2001) 177-181. 
[28] M.E. Meyer, D.W. Swanepoel, T. Le Roux, M. van der Linde. Early detection of infant hearing loss in the private health care sector of South Africa. Int. J. Pediatr. Otorhinolaryngol. 76 (2012) 698-703.

[29] J.E. Kerschner, Neonatal hearing screening: to do or not to do. Pediatr Clin N Am. 51 (2004) 725-736.

[30] S. Meier, O. Narabayashi, R. Probst, N. Schmuziger, N. Comparison of currently available devices designed for newborn hearing screening using automated auditory brainstem and/or otoacoustic emission measurements. Int. J. Pediatr. Otorhinolaryngol. 68 (2004) 927-934.

[31] A. Melagrana, S. Casale, M.G. Calevo, V. Tarantino. MB11 BERAphone and auditory brainstem response in newborns at audiologic risk: comparison of results. Int. J. Pediatr. Otorhinolaryngol. 71 (2007) 1175-1180.

[32] Maico Diagnostic GmbH. Device for determining acoustically evoked brainstem potentials, http://www.maico-diagnostic.de, 2007 (accessed 23 February 2014).

[33] Western Cape Government @ 2014. September 2013, http://www.westerncape.gov.za/service/post-natal-after-birth-health-care, (accessed 28 June 2014).

[34] M. Theunissen, D. Swanepoel, Early hearing detection and intervention services in the public health sector of South Africa, Int. J. Audiol. 47 (2008) S23-S29.

[35] M. Cebulla, H. Sofia, S. Wafaa. Sensitivity of ABR based newborn screening with the MB11 BERAphone $<$ sup $>\circledR</$ sup $>$. Int. J. Pediatr. Otorhinolaryngol. 78 (2014) 756761. 
[36] J.I. Benito-Orejas, B. Ramírez, D. Morais, A. Almaraz, J.L. Fernández-Calvo, Comparison of two-step transient evoked otoacoustic emissions (TEOAE) and automated auditory brainstem response (AABR) for universal newborn hearing screening programs. Int. J. Pediatr. Otorhinolaryngol. 72 (2008) 1193-1201.

[37] O. Konukseven, A. Genc, T. Muderris, M.K. Kayikci, D. Turkyilmaz, B. Ozturk, B. Gunduz, Can Automated Auditory Brainstem Response be Used as an Initial Stage Screening Test in Newborn Hearing Screening Programs?. Int. Adv. Otol 6 (2010) 231238.

[38] B. Vos, R. Lagasse, A. Levêque. Main outcomes of a newborn hearing screening program in Belgium over six years. Int. J. Pediatr. Otorhinolaryngol. 78 (2014) 14961502.

[39] S. Bansal, A. Gupta, A. Nagarkar, Transient evoked otoacoustic emissions in hearing screening programs-Protocol for developing countries. Int. J. Pediatr. Otorhinolaryngol. 72 (2008) 1059-1063.

[40] B.O. Olusanya, Making targeted screening for infant hearing loss an effective option in less developed countries. . Int. J. Pediatr. Otorhinolaryngol. 75 (2011) 316321.

[41] A. Pisacane, G. Auletta, F. Toscano, M. Errichiello, F. Barrier, P. Riccardi, Carla Laria et al. Feasibility and effectiveness of a population-based newborn hearing screening in an economically deprived region of Italy. . Int. J. Pediatr. Otorhinolaryngol. 77 (2013) 329-333.

[42] T. Smolkin, O. Mick, M. Dabbah, S. Blazer, G. Grakovsky, N. Gabay, A. Gordin, I.R. Makhoul. Birth by Cesarean delivery and failure on first otoacoustic emissions hearing test. Pediatrics 130 (2012) 95-100. 
[43] A.M. Augustine, A.K. Jana, K.A. Kuruvilla, S. Danda, A. Lepcha, J. Ebenezer, R.R. Paul, A. Tyagi, A. Balraj, Neonatal hearing screening-Experience from a tertiary care hospital in Southern India. Indian pediatrics 51 (2014) 179-183.

[44] L. Gibbons, J.M. Belizán, J. A. Lauer, A.P. Betrán, M. Merialdi, F. Althabe, The global numbers and costs of additionally needed and unnecessary caesarean sections performed per year: overuse as a barrier to universal coverage, World health report 30 (2010) 1-31.

[45] L.J. Scheepers, D.W. Swanepoel, T. le Roux. Why parents refuse newborn hearing screening and default on follow-up rescreening-A South African perspective. . Int. J. Pediatr. Otorhinolaryngol. 78 (2014) 652-658.

[46] B.O. Olusanya, S. L. Wirz, L. M. Luxon. Hospital-based universal newborn hearing screening for early detection of permanent congenital hearing loss in Lagos, Nigeria. . Int. J. Pediatr. Otorhinolaryngol. 72 (2008) 991-1001.

[47] N. Friderichs, D.W. Swanepoel, J.W. Hall III. Efficacy of a community-based infant hearing screening program utilizing existing clinic personnel in Western Cape, South Africa. Int. J. Pediatr. Otorhinolaryngol. 76 (2012) 552-559.

[48] B.O. Olusanya, S.L. Wirz, L.M. Luxon, Community-based infant hearing screening for early detection of permanent hearing loss in Lagos, Nigeria: a cross-sectional study, Bull. World Health Organ. 86 (2008) 956-963. 